Check for updates

Cite this: Phys. Chem. Chem. Phys., 2018, 20, 10193

Received 13th December 2017, Accepted 21st March 2018

DOI: $10.1039 / c 7 c p 08349 a$

rsc.li/pccp

\title{
Rheology of phosphonium ionic liquids: a molecular dynamics and experimental study
}

\author{
Sten Sarman, (D) *a Yong-Lei Wang, (D) ab Patrick Rohlmann, (D) ${ }^{c}$ \\ Sergei Glavatskih (D) ${ }^{c d}$ and Aatto Laaksonen (D) ${ }^{\text {ae }}$
}

\begin{abstract}
We have studied the rheological behavior of the ionic liquid trihexyl(tetradecyl)phosphonium bis(mandelato)borate, $\left[\mathrm{P}_{66614}\right][\mathrm{BMB}]$, and compared it with that of another ionic liquid, namely trihexyl(tetradecyl)phosphonium chloride, $\left[\mathrm{P}_{66614}\right][\mathrm{Cl}]$. The non-halogenated $\left[\mathrm{P}_{66614}\right][\mathrm{BMB}]$ has been selected as it is known to provide enhanced lubrication performance and is, consequently, of technological importance. The ionic liquid $\left[\mathrm{P}_{66614}\right][\mathrm{Cl}]$, despite its relatively simple anion, exhibits viscosities very similar to those of $\left[\mathrm{P}_{66614}\right][\mathrm{BMB}]$, making it an excellent reference fluid for the modeling study. The viscosities of the ionic liquids have been obtained by equilibrium atomistic simulations using the Green-Kubo relation, and by performing nonequilibrium shear flow simulations. The influence of the simulation system size and a reduction of the atomic charges on the viscosities of the ionic liquids are systematically studied. The atomic charges are reduced to mimic the temperature dependent charge transfer and polarization effects. It has been found that scaling the point charges with factors between 0.60 and 0.80 from full ion charges can provide reliable viscosities of $\left[\mathrm{P}_{66614}\right][\mathrm{BMB}]$, consistent with the experimentally measured viscosities within the studied temperature interval from 373 to $463 \mathrm{~K}$. The viscosities of $\left[\mathrm{P}_{66614}\right][\mathrm{Cl}]$ have been obtained with scaling factors between 0.80 and 1.0 reflecting the lower polarizability and charge transfer effects of the chloride anion.
\end{abstract}

\section{Introduction}

Ionic liquids (ILs) are rather complex molecular salts composed of bulky, asymmetric and flexible organic molecular cations and weakly coordinating organic or inorganic anions. Their melting points are low, i.e. $100{ }^{\circ} \mathrm{C}$ or less, due to low lattice energies and high (conformational) entropies. The ionic components interact via both strong long-ranged Coulombic forces and short-ranged van der Waals forces. The conformational flexibility of the sizable cations can be very large and voluminous. They do not always exist as pure isotropic homogeneous liquids but rather show distinct heterogeneous local ordering such as micellar or smectic phases. $^{1,2}$ Their interactions with themselves and with different materials and nanoparticles are highly complex. The dynamic behavior of the ILs is slow and heterogeneous, ${ }^{3,4}$ often containing several motional modes, ${ }^{5,6}$ which leads to long relaxation times.

\footnotetext{
${ }^{a}$ Department of Materials and Environmental Chemistry, Arrhenius Laboratory, Stockholm University, 10691 Stockholm, Sweden. E-mail: sarman@ownit.nu

${ }^{b}$ Department of Chemistry, Stanford University, Stanford, California 94305, USA

${ }^{c}$ Department of Machine Design, Royal Institute of Technology, 10044 Stockholm, Sweden

${ }^{d}$ Department of Electrical Energy, Metals, Mechanical Constructions and Systems, Ghent University, Ghent, 9000, Belgium

${ }^{e}$ Department of Chemistry, Ångström Laboratory, Uppsala University, Box 538, 75121 Uppsala, Sweden
}

Their structure and dynamics change easily with temperature, concentration, co-solvents and $\mathrm{pH}$ value. Therefore, the ILs are molecular systems that are difficult to study, both experimentally and theoretically. ${ }^{7}$

The ionic nature of the ILs makes them very attractive candidates for many energy related applications ${ }^{8}$ such as fuel and solar cells, supercapacitors and high-energy electrochemical devices. Lubrication is another key application, where the ILs have the potential to significantly enhance the energy efficiency. Unlike conventional lubricants, the ionic systems are electrically conductive and may provide more efficient interactions with the lubricated metal surfaces, which are usually electron deficient as a result of rubbing. Anions move to the positively charged surfaces forming boundary films capable of reducing friction and wear. This also creates a possibility of active friction control $^{9-11}$ by changing the surface charge and the boundary layer structure and composition. ${ }^{12}$ Ion dynamics depends on the IL viscosity but it remains unclear whether or not it is possible to control the $\mathrm{IL}$ rheology by an electric field. ${ }^{13}$ It is, however, possible to significantly vary the viscosity of the IL by proper selection of the cations and anions. A great advantage of the ILs is their inherent functional tunability, based on the large number of possibilities to combine the cations with different organic moieties with different anions. At the same time, the ions can be further functionalized by changing specific atoms in the constituent cations or anions. 
So far many studies have been undertaken on, among other substances, tetrafluoroborate and hexafluorophosphate anions. ${ }^{14}$ However, these halogen-containing ions react easily with water leading to corrosion due to formation of hydrogen halides. Therefore, non-halogenated ILs have been preferred in later studies on lubrication applications. ${ }^{15-17}$ Since many non-halogenated phosphorus and boron containing ILs show low friction and wear performance and thus are of technological importance, we have selected the IL trihexyl(tetradecyl)phosphonium bis(mandelato)borate, $\left[\mathrm{P}_{66614}\right][\mathrm{BMB}]$, for our study.

A number of works on the rheology and viscosity of ILs have been reported during the last fifteen years. ${ }^{18-36}$ In most cases, the ILs have been composed of a cation consisting of an imidazolium based core substituted with alkyl chains. These cations have been combined with a wide range of anions such as tetrafluoroborate, hexafluorophosphate, methylsulfate, trifluoromethylsulfonate, acetate, trifluoroacetate, and bis(trifluoromethylsulfonyl)amide. There is also one work where the imidazolium core has been replaced by other heterocyclic rings, for example, pyrrolidine, pyridine, piperidine, morpholine, ammonium and phosphonium. ${ }^{21}$ Another study has reported modeling results for a liquid composed of 1,3-dimethylimidazolium cations and bis(trifluoromethylsulfonyl)imide anions. ${ }^{37}$ The viscosities have been evaluated by equilibrium Green-Kubo relations, the Müller Plathe method and by direct simulation of a liquid in a narrow slit with walls consisting of FeO. These methods yielded the same equilibrium viscosity and the two latter methods gave consistent results in the nonNewtonian shear thinning regime, even though image charges were not taken into account.

However, the molecules, that we intend to study in the present work with an alkylated phosphonium cation and a substituted borate anion, are considerably larger, whereby the relaxation times become much longer, so that appreciably longer simulations are needed. Moreover, in order to ascertain that the estimates of the viscosities are correct we are going to evaluate them both by Green-Kubo relations and by performing shear flow simulations using the SLLOD equations of motion. According to linear response theory, these two methods should give exactly the same results in the linear or Newtonian regime. To provide further insight in the structure-viscosity correlations, the IL trihexyl(tetradecyl)phosphonium chloride, $\left[\mathrm{P}_{66614}\right][\mathrm{Cl}]$ has been selected for comparison as it exhibits similar viscosities as $\left[\mathrm{P}_{66614}\right][\mathrm{BMB}]$ despite the significantly smaller anion.

\section{Theory, ionic model, simulation methodology, and measurement procedure}

\subsection{Simulation algorithms for the viscosity}

There are a few different methods available for the evaluation of the viscosity. They include equilibrium methods such as the Green-Kubo relation ${ }^{20,24,25,31-33,35,36}$ and the corresponding Einstein relation, ${ }^{21-23,29,34}$ i.e. the integrated Green-Kubo relation, and non-equilibrium methods such as direct shear flow simulations using the SLLOD equation of motion, ${ }^{21}$ sinusoidal velocity profiles ${ }^{18}$ and the Müller-Plathe method. ${ }^{26-28,30}$ These methods have been used on a large scale to evaluate the viscosity of nonpolar models of alkanes ${ }^{38,39}$ and they have also been generalised to ILs. ${ }^{21,23,27}$ So far they have predominantly been applied to imidazolium-based compounds with a comparatively low viscosities and short relaxation times. In this work, on the other hand, we intend to evaluate the viscosity of compounds based on tetraalkylphosphonium since they, among other useful properties, show very good lubricating performance. However, these molecules are much larger and have considerably longer relaxation times than previously studied molecules. Therefore, larger computational resources are needed and special attention must be paid to the simulations algorithms used to evaluate the viscosities.

More specifically, we are going to apply the Green-Kubo relation because it gives the exact viscosity in the linear or Newtonian regime and it is easy to implement in an existing molecular dynamics simulation code. The only drawback is that the time correlation functions of the shear stresses of the ILs converge very slowly so that much longer simulations are needed to evaluate their viscosities compared to those of Lennard-Jones liquids or of nonpolar alkanes. The Green-Kubo relation for the viscosity can be expressed as:

$$
\eta=\frac{V}{10 k_{\mathrm{B}} T} \int_{0}^{\infty} \mathrm{d} t\left\langle\overline{\boldsymbol{\sigma}}^{s}(t): \overline{\boldsymbol{\sigma}}^{s}(0)\right\rangle_{\mathrm{eq}},
$$

where $V$ is the volume of the system, $k_{\mathrm{B}}$ is Boltzmann's constant, $T$ is the absolute temperature, $\overline{\boldsymbol{\sigma}}^{s}=(1 / 2)\left(\boldsymbol{\sigma}+\boldsymbol{\sigma}^{T}\right)-$ $(1 / 3) \operatorname{Tr}(\boldsymbol{\sigma})$ is the symmetric traceless part of the stress tensor and the subscript 'eq' denotes that the average is evaluated in an equilibrium ensemble. Note that the time correlation functions of the normal stress differences, which are equivalent to shear stresses, are included in this integral. This gives another two independent signals that reduce the statistical noise.

Since the relaxation times are very long it could be difficult to determine whether the Green-Kubo integrals have converged. Therefore, we will cross-check the results by performing shear flow simulations applying the SLLOD equations of motion. ${ }^{40,41}$ Then a streaming velocity, $\mathbf{u}=\dot{\gamma} z \mathbf{e}_{x}$, i.e. a velocity in the $x$-direction varying linearly in the $z$-direction, is added to the ordinary Newtonian equations of motion,

$$
\dot{\mathbf{q}}_{i \alpha}=\frac{\mathbf{p}_{i \alpha}}{m_{\alpha}}+\dot{\gamma} \mathbf{e}_{x} z_{i \alpha}
$$

and

$$
\dot{\mathbf{p}}_{i \alpha}=\mathbf{F}_{i \alpha}-\dot{\gamma} \mathbf{e}_{x} p_{i \alpha z}-\zeta \mathbf{p}_{i \alpha},
$$

where $\mathbf{p}_{i \alpha}$ and $\mathbf{q}_{i \alpha}$ are the linear momentum relative to the streaming velocity and the position of atom $\alpha$ of molecule $i, \dot{\gamma}$ is the velocity gradient or shear rate, $m_{\alpha}$ is the mass of atom $\alpha$, and $\zeta$ is a thermostatting multiplier removing the excess heat generated by the shear field. The algebraic expressions for this multiplier can be selected in a few different ways depending on the desired ensemble. These eqn (2a) and (2b) drive a shear flow, which is similar to the flow in a macroscopic viscometer, 
so we actually have a theoretical viscometer. By applying the Lees-Edwards sliding brick boundary conditions ${ }^{41}$ the edge effects vanish and it is actually a small homogeneous volume of shearing liquid in a large surrounding homogeneous volume of shearing liquid that is simulated. The viscosity, $\eta$, is obtained as the ratio of the shear stress, $\sigma_{z x}{ }^{s}$, and the velocity gradient,

$$
\eta=\lim _{t \rightarrow \infty} \lim _{\dot{\gamma} \rightarrow 0}\left\langle\sigma_{z x}^{s} / \dot{\gamma}\right\rangle .
$$

By using linear response theory it can be proven that this relation gives exactly the same result for the viscosity as the Green-Kubo relation (eqn (1)) in the limit of zero shear rate, so that an important cross-check is provided. Moreover, the SLLOD equations of motion are an exact description of adiabatic planar Couette flow and they are also a good approximation of non-Newtonian flow at high shear rates.

\subsection{Ionic models, polarization and charge transfer effects}

The molecular structures of the studied ILs, trihexyl(tetradecyl)phosphonium bis(mandelato)borate, $\left[\mathrm{P}_{66614}\right][\mathrm{BMB}]$, and trihexyl(tetradecyl)phosphonium chloride $\left[\mathrm{P}_{66614}\right][\mathrm{Cl}]$, are shown in Fig. 1. The ionic groups are represented by an atomistic force field model where the atoms in the molecules are treated like Lennard-Jones interaction sites decorated with full or partial electrostatic charges and the intramolecular interactions include bond stretching; covalent angle bending and torsional angle potentials. The cross-interaction parameters between unlike atoms are given

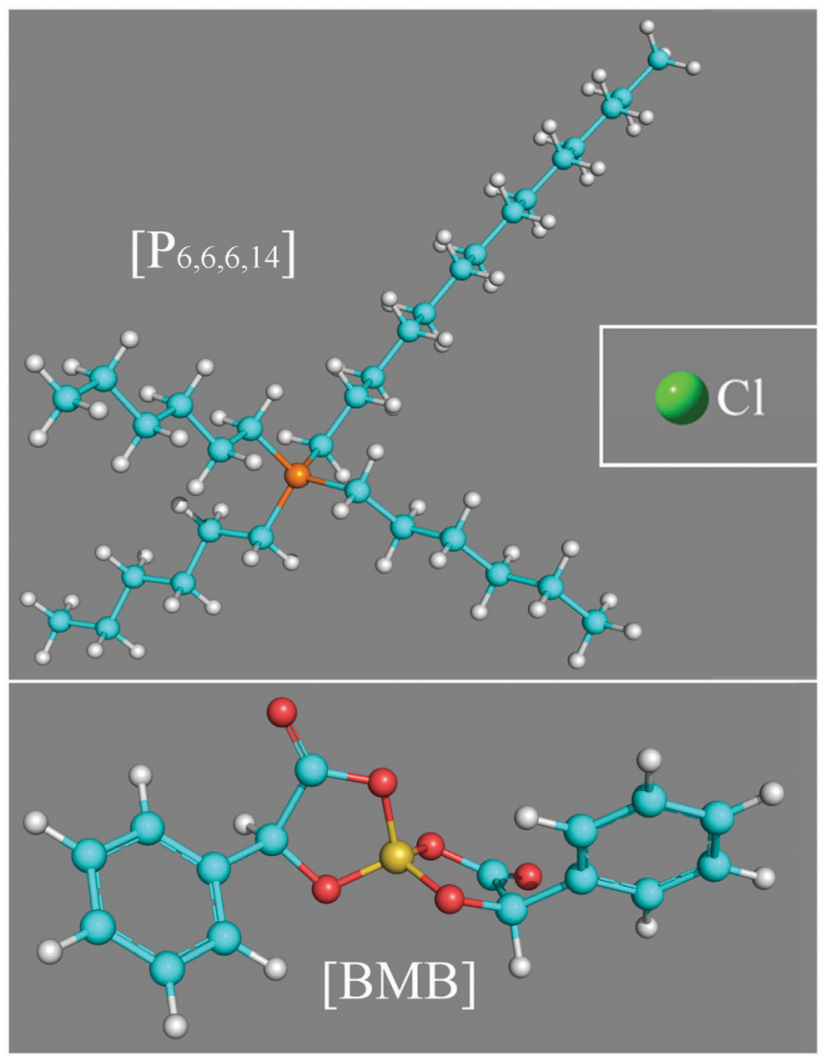

Fig. 1 Molecular structures of the $\left[\mathrm{P}_{66614}\right]^{+}$cation, $[\mathrm{BMB}]^{-}$anion and $[\mathrm{Cl}]^{-}$ anion studied in this work. by the Lorentz-Berthelot combining rules. The actual numerical values of the atomistic force field parameters for the $\left[\mathrm{P}_{66614}\right]^{+}$ cation and $[\mathrm{BMB}]^{-}$, and $[\mathrm{Cl}]^{-}$anions were taken from a systematically developed force field in our previous works, ${ }^{6,42}$ where both inter- and intra-molecular interaction parameters were refined to achieve a quantitative description of the intermolecular ionic structures obtained from quantum chemistry $a b$ initio calculations, and adapted to fit vibration frequency data derived from experimentally measured data. ${ }^{16}$

Special attention is paid to the atomic partial charges since they are highly important parameters but there is no unique way of determining them as they are not quantum mechanical observables. In the case of ILs it was observed already in the early simulations ${ }^{43,44}$ that keeping full \pm 1.0 charges for the ionic species would, in most cases, produce a far too viscous liquid where the molecules diffuse too slowly. This is due to the intrinsic nature of the bulky molecular ions getting easily polarized and also allowing a certain degree of charge transfer between the ions when they are close to each other, so that the ion charges are spread out. This problem could in principle be handled by applying an ab initio simulation scheme where the electrons adapt to arbitrary configurations including those with a high degree of structural heterogeneity. Unfortunately, it is not feasible to apply this method in practice because it requires vast computational resources. A more approximate but less computationally demanding approach is to treat the polarizability explicitly in the atomic potentials used in atomistic simulations by using polarizable force fields. ${ }^{19,21,45}$ There are several ways to determine the atomic polarizability. The designed regression and similar statistical approaches can predict the atomic polarizability based on the molecular topology assuming that the polarizabilities of the neighboring atoms do not interact with the target atom. ${ }^{46}$ In more sophisticated methods the atomic coordinates are used to compute averaged polarizabilities for important chemical elements including the influence from vicinal atoms via dipole-dipole tensors, ${ }^{47,48}$ or the distributed polarizability model $^{49}$ and a general automatic atomic model parametrization procedure $^{50}$ could be used. However, even this simpler method is computationally too demanding for the complex ILs considered in this study, so it cannot be used for the evaluation of the viscosity. Fortunately, there is one last remaining approximate solution to estimate the effect of polarization and charge transfer, namely by scaling or reducing the electrostatic point charges decorating the Lennard-Jones interaction sites representing the atoms in the molecules. This downscaling of the partial charges is justified by the fact that the net charges of the cations and anions yield fractional values in quantum chemical calculations of single ion pairs. ${ }^{51}$ In principle, the fractional charges in quantum chemical calculations of single ion pairs arise from the overlap of diffusive wave-functions and subsequent assignment of electron density to the cationic/anionic part based on the algorithm of partial charge methods, such as ChelpG and the restraint electrostatic potential fitting approach. ${ }^{52,53}$ In the simplest case, an ad hoc scaling of all the charges is performed which often results in a good agreement with experiments. A more rigorous scaling of charges can be achieved by taking liquid configurations from atomistic simulations to calculate 
a new set of charges in a repeated procedure until some form of self-consistency is reached. ${ }^{44}$ Although the changes in the thermodynamic and structural properties are normally very small upon adjusting the charges, the effects on the transport properties are often significant.

In most simulation studies of ILs with atomistic force fields, where a uniform charge reduction was done, the scaling of charges to roughly 0.80 did work very well to shift the diffusion coefficients reasonably close to the experimental values at some parts of the temperature intervals considered in the investigations. ${ }^{54,55}$ Clearly this is a very approximate solution to the problem. First, scaling all the charges uniformly is an ad hoc procedure. Secondly, there are all the other force field parameters originally adjusted together with the rigid point charges. Thirdly, the factor around 0.80 is by no means universal even if it appears to have been working in many of the most common and simpler ILs. Fourthly, the charge polarizability of the ions may depend on the temperature and thus the scaling factor too.

\subsection{Atomistic simulation details}

Atomistic simulations were performed by using the GROMACS 5.0.4 package ${ }^{56}$ with standard cubic periodic boundary conditions in the equilibrium simulations and with Lees-Edwards sliding brick boundary conditions in the non-equilibrium shear flow simulations. The equations of motion were integrated using the velocity Verlet algorithm with a time step of 0.5 fs. The cutoff distance of the van der Waals interactions and the electrostatic interactions in real space between the atomcentered point charges was set to $1.44 \mathrm{~nm}$. The Particle-Mesh Ewald method was used to handle the long-range electrostatic interactions in reciprocal space with a grid spacing of $0.12 \mathrm{~nm}$ and an accuracy of $10^{-6}$. The bulk IL simulation system was first energetically minimized using the steepest descent method, and then annealed gradually from $1200 \mathrm{~K}$ down to the target temperatures within 10 ns maintained using a NoséHoover thermostat and a Parrinello-Rahman barostat under a pressure of 1 atmosphere with time coupling constants of 200 and 500 fs, respectively. Thereafter, all the IL simulation systems were equilibrated for $50 \mathrm{~ns}$ before the production runs. Most of the simulations of $\left[\mathrm{P}_{66614}\right][\mathrm{BMB}]$ were carried out for a system consisting of 96 ion pairs with a time-step of 0.5 fs, whereas 256 ion pairs and a time step of 1.0 fs were used for $\left[\mathrm{P}_{66614}\right][\mathrm{Cl}]$. At each state point 8 parallel simulations from different start configurations were independently performed and averaged during $400 \mathrm{~ns}$. The upper limit in the Green-Kubo integral was $8 \mathrm{~ns}$. This gave a relative error of about $10 \%$ or less in the viscosities. In order to examine the system size effects, some simulations of $\left[\mathrm{P}_{66614}\right][\mathrm{BMB}]$ were performed with 735 ion pairs with 20 parallel simulations from different starting configurations with a length of $160 \mathrm{~ns}$ each. We also performed a simulation of a large system consisting of 6000 ion pairs along a single trajectory of a length of $120 \mathrm{~ns}$.

\subsection{Ionic liquids and experimental measurement procedure}

Trihexyl(tetradecyl)phosphonium bis(mandelato)borate, $\left[\mathrm{P}_{66614}\right]$ [BMB], was synthesized as previously reported. ${ }^{16}$ The water content was determined by an indirect Karl Fischer titration method after drying the IL in a vacuum oven for 72 hours at $333 \mathrm{~K}$. The measured water contents of $\left[\mathrm{P}_{66614}\right][\mathrm{BMB}]$ was 0.1 percent by weight. The IL $\left[\mathrm{P}_{66614}\right][\mathrm{Cl}]$, purchased from Cytec Industries Inc., was used as received. The rheological measurements were performed with HR2 and HR3 rheometers (TA Instruments, USA). A parallel plate configuration, with a stainless steel upper plate of $20 \mathrm{~mm}$ in diameter, was used. A Peltier element, located under the bottom stainless steel plate, made it possible to actively control the temperature of the tested liquid. The rheometers were calibrated using a calibration oil. Prior to each measurement, the plates were cleaned with isopropanol and ethanol and then dried under air flow. The viscosity measurements were conducted over a temperature range from $323 \mathrm{~K}$ to $473 \mathrm{~K}$ with steps of $10 \mathrm{~K}$.

\section{Results and discussion}

Since the viscosity of a complex system, such as the tetraalkylphosphonium based ILs, has never, to our best knowledge, been evaluated by atomistic simulations, we first evaluate the dependence of the viscosity of $\left[\mathrm{P}_{66614}\right][\mathrm{BMB}]$ on the simulation system size, and verify that a proper simulation system is used to obtain reliable computed viscosities. The effect of the charge scaling factors on the viscosities of $\left[\mathrm{P}_{66614}\right][\mathrm{BMB}]$ and $\left[\mathrm{P}_{66614}\right][\mathrm{Cl}]$ are systematically studied at different temperatures, and the computed viscosities are compared with the experimentally measured viscosities. The computed viscosities, estimated from nonequilibrium shear flow simulations, are also compared with those obtained from equilibrium simulations using the GreenKubo relation as well as with the experimentally measured viscosities.

\subsection{System size effect}

The values of many thermodynamic, structural and transport properties estimated by molecular dynamics simulations may be dependent ${ }^{57}$ on the size of the simulated system. Thus, it is desirable to use a large system not least for the ILs due to their long range correlations and inherently heterogeneous structure and dynamics. ${ }^{58,59}$ However, the fluctuations decrease with the system size, which means that more memory and longer computation times are needed to evaluate the Green-Kubo relations for the shear viscosity. Therefore, it is desirable to use a small system. In order to determine the smallest system size that still yields reliable results it is suitable to perform exploratory simulations of a few systems of different sizes and to compare the results.

In the present work three simulation systems composed of 96,735 and $6000\left[\mathrm{P}_{66614}\right][\mathrm{BMB}]$ ion pairs, respectively, were constructed, and the corresponding shear viscosities were calculated at different temperatures using the Green-Kubo relation and shear flow simulations. The obtained simulation results are shown in Tables 1 and 3. The shear viscosities for $\left[\mathrm{P}_{66614}\right][\mathrm{BMB}]$ estimated from the small simulation system consisting of 96 ion pairs with a charge scaling factor, $s$, of either 0.80 or 0.60 at temperatures between 423 and $463 \mathrm{~K}$, are 
Table 1 Dependence of the viscosity of $\left[\mathrm{P}_{66614}\right][\mathrm{BMB}]$ on the size of the simulation system consisting of different numbers $(N)$ of ion pairs. These computed viscosities are obtained from equilibrium atomistic simulations using Green-Kubo relation at different temperatures ( $T$ ) with varied charge scaling factors (s), and compared with the experimentally measured viscosity values

\begin{tabular}{|c|c|c|c|c|c|}
\hline$T / \mathrm{K}$ & $N=96, s=0.60, \eta / \mathrm{mPa} \mathrm{s}$ & $N=96, s=0.80, \eta / \mathrm{mPa} \mathrm{s}$ & $N=735, s=0.60, \eta / \mathrm{mPa} \mathrm{s}$ & $N=735, s=0.80, \eta / \mathrm{mPa} \mathrm{s}$ & Measured, $\eta / \mathrm{mPa} \mathrm{s}$ \\
\hline 373 & $75 \pm 10$ & $180 \pm 20$ & & & 67 \\
\hline 383 & $68 \pm 2$ & $162 \pm 6$ & & & 47 \\
\hline 403 & $29 \pm 2$ & $67 \pm 2$ & & & 27 \\
\hline 423 & $16 \pm 1$ & $33 \pm 1$ & $16 \pm 2$ & $30 \pm 3$ & 18 \\
\hline 443 & $12 \pm 1$ & $20 \pm 1$ & & $16 \pm 2$ & 14 \\
\hline 463 & $6 \pm 1$ & $10 \pm 0.5$ & & $10 \pm 1$ & 11 \\
\hline
\end{tabular}

consistent with those obtained from the large simulation system composed of 735 ion pairs at the same temperature and with the same charge scaling factors. The Green-Kubo relations do not converge for the largest system but the viscosities obtained by the shear flow simulations agree with those of the smaller systems.

These calculations do not necessarily prove that there is no system size dependence of the shear viscosities of $\left[\mathrm{P}_{66614}\right][\mathrm{BMB}]$, since the systems studied are still small compared to macroscopic systems. However, the results for the systems of 96 ion pairs agree within relative errors of 15 percent or less with the results for the system of 735 ion pairs and within 25 percent or less with the results of the largest system. The viscosities at the shear rates of $97.7 \times 10^{6}$ and $19.5 \times 10^{6} \mathrm{~s}^{-1}$ are about 20 percent lower in the largest system than in the two smaller systems. This is partly due to system size dependence and partly due to the fact that the onset of shear thinning may take place at slightly lower shear rates in larger systems. Therefore, the error is not too large if we use 96 ion pairs, which have been used unless otherwise mentioned. It should be noted there are some recent works ${ }^{35,36}$ where reasonably accurate viscosities are obtained by using systems of 200-300 ion pairs. Finally, it should be noted that the charge scaling factors, used to compensate for the polarizability and the charge transfer in the present work and in the mentioned references to some extent compensate for the system size dependence as well.

\subsection{Charge scaling factors}

It has been shown in earlier works that downscaling the partial charges on the atoms is an effective way to account for polarization effects and charge transfer among molecular ions and thereby improve the reliability of the calculations of transport coefficients and other dynamic properties of the modeled IL systems. ${ }^{44,54,55}$ Herein we consider two charge scaling factors, $s=0.80$ and 0.60 , for $\left[\mathrm{P}_{66614}\right][\mathrm{BMB}]$. For $\left[\mathrm{P}_{66614}\right][\mathrm{Cl}]$ a charge scaling factor of 0.80 and the standard unity ion charge $( \pm 1.0)$ are used to calculate its viscosity at different temperatures as a reference.

In Fig. 2 we show the shear viscosities of $\left[\mathrm{P}_{66614}\right][\mathrm{BMB}]$ calculated from equilibrium molecular dynamics simulations using the two different atomic charge scaling factors and the corresponding experimentally measured values. The measured and computed viscosities are also shown in Table 1 . The computed viscosities of $\left[\mathrm{P}_{66614}\right][\mathrm{BMB}]$ follow the empirical Arrhenius equation $\eta=\eta_{0} \exp \left(-E_{\mathrm{a}} / R T\right)$, where $\eta_{0}$ is a preexponential factor, $R$ is the gas constant, $T$ is the absolute temperature, and $E_{\mathrm{a}}$ can be thought of as an activation energy

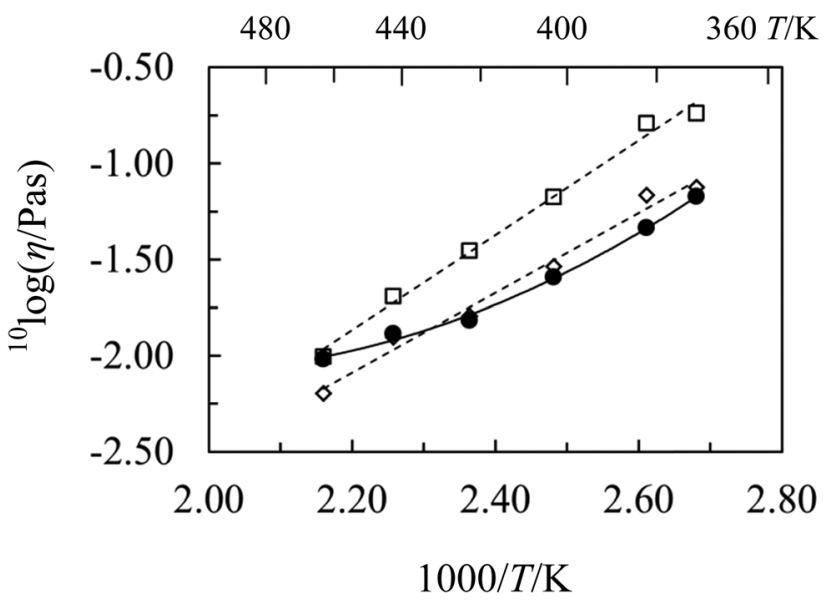

Fig. 2 Computed viscosities of $\left[\mathrm{P}_{66614}\right][\mathrm{BMB}]$ obtained from equilibrium atomistic simulations of a system consisting of 96 ion pairs using the Green-Kubo relation as a function of the inverse temperature with charge scaling factors of 0.80 (open squares) and of 0.60 (open diamonds). The computed viscosities are compared with the experimentally measured viscosities (filled circles).

for viscous flow. At the same time, it can be observed that the experimentally measured viscosities deviate from the Arrhenius equation. The deviation is apparently due to the polarization and charge transfer effects in the ILs and they can be accounted for by the scaling factors. The shear viscosity of $\left[\mathrm{P}_{66614}\right][\mathrm{BMB}]$ approximately doubles at the same temperature, when the charge scaling factor increases from 0.60 to 0.80 . In the temperature range $373-443 \mathrm{~K}$, the experimentally measured viscosities agree reasonably well with the computed viscosities obtained with a charge scaling factor of 0.60 . However, this changes at the highest temperature of $463 \mathrm{~K}$, where a charge scaling factor of 0.80 is needed to reproduce the experimentally measured viscosity of $\left[\mathrm{P}_{66614}\right][\mathrm{BMB}]$.

Qualitatively similar tendencies, for both the temperature dependence of the viscosity and the effect of the charge scaling factor on the viscosity, are observed in the $\left[\mathrm{P}_{66614}\right][\mathrm{Cl}]$ simulations, as shown in Table 2 and Fig. 3. The experimentally measured viscosities fall in between the computed viscosities with charge scaling factors of 1.0 and 0.80 . Once again, the computed viscosities follow the Arrhenius-like expression, whereas the experimentally measured viscosities do not.

It is noteworthy that different, i.e. smaller, charge scaling factors are needed for $\left[\mathrm{P}_{66614}\right][\mathrm{BMB}]$ than for $\left[\mathrm{P}_{66614}\right][\mathrm{Cl}]$ to obtain computed viscosities in agreement with the experimentally 
Table 2 Dependence of the viscosity of $\left[\mathrm{P}_{66614}\right][\mathrm{Cl}]$ on the charge scaling factors. These computed viscosities are obtained from equilibrium atomistic simulations of a system consisting of $256\left[\mathrm{P}_{66614}\right][\mathrm{Cl}]$ ion pairs using the Green-Kubo relation at different temperatures, and compared with the experimentally measured viscosity values

\begin{tabular}{lccc}
\hline$T / \mathrm{K}$ & $\eta / \mathrm{mPa} \mathrm{s}(s=0.80)$ & $\eta / \mathrm{mPa} \mathrm{s}(s=1.0)$ & Measured, $\eta / \mathrm{mPa} \mathrm{s}$ \\
\hline 323 & $300 \pm 20$ & & 402 \\
343 & $130 \pm 7$ & $320 \pm 20$ & 167 \\
363 & $62 \pm 3$ & $146 \pm 11$ & 86 \\
383 & $31 \pm 1$ & $66 \pm 5$ & 48 \\
403 & $13.5 \pm 0.4$ & $30 \pm 1$ & 29 \\
423 & $9.5 \pm 0.6$ & $22 \pm 2$ & 18 \\
463 & $4.6 \pm 0.2$ & & 9
\end{tabular}

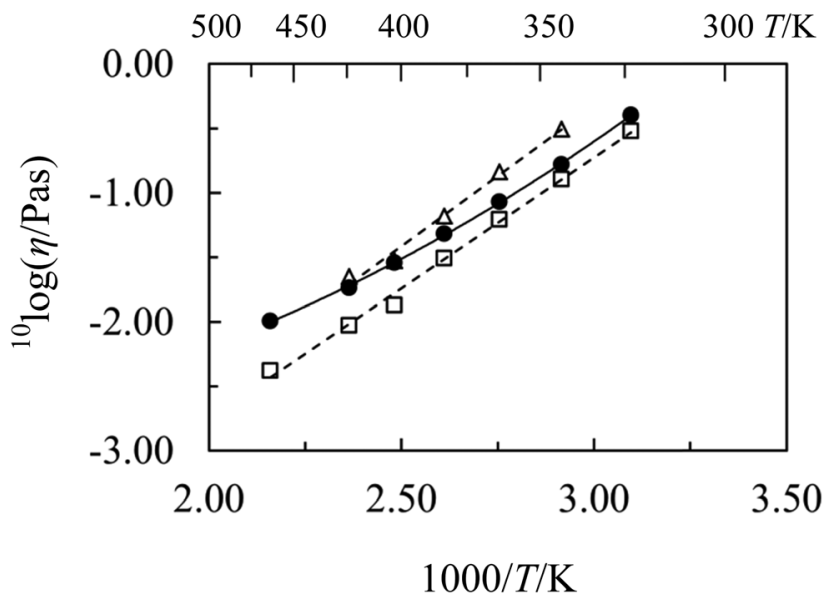

Fig. 3 Computed viscosities of $\left[\mathrm{P}_{66614}\right][\mathrm{Cl}]$ obtained from equilibrium atomistic simulations of a system consisting of 256 ion pairs using the Green-Kubo relation as a function of the inverse temperature with charge scaling factors of 1.0 (open triangles) and 0.80 (open squares). These computed viscosities are compared with the experimentally measured viscosities (filled circles). measured viscosities. This is most likely due to intrinsic differences in both the size and the structure of the $[\mathrm{Cl}]^{-}$and $[\mathrm{BMB}]^{-}$ anions (mono-atomic vs. bulky molecular). Compared with the monoatomic $[\mathrm{Cl}]^{-}$anion, the $[\mathrm{BMB}]^{-}$anion is much larger and characterized by distinct orientational distributions of carbonyl and phenyl rings in the $[\mathrm{BMB}]^{-}$anionic framework. Such an ionic structure indicates that the charges (electrons or lack of electrons) in the $[\mathrm{BMB}]^{-}$anionic framework are delocalized and the polarizability of the $[\mathrm{BMB}]^{-}$anion is stronger than that for the relatively small $[\mathrm{Cl}]^{-}$anion. These molecular features should be enough to promote the distinct charge transfer between the $[\mathrm{BMB}]^{-}$anions and the neighboring $\left[\mathrm{P}_{66614}\right]^{+}$cations, and thus the $[\mathrm{BMB}]^{-}$anions have smaller effective charges than the $[\mathrm{Cl}]^{-}$anions. So far it has been assumed that the scaling factors compensate for the polarizability and charge transfer but it should be noted that they may also compensate for the system size dependence.

\subsection{Radial distribution functions}

Further insight into the relation between the viscosities and the structural properties of $\left[\mathrm{P}_{66614}\right][\mathrm{BMB}]$ and $\left[\mathrm{P}_{66614}\right][\mathrm{Cl}]$ can be gained by analyzing the radial distribution functions (RDFs) between the $\left[\mathrm{P}_{66614}\right]^{+}$cations and the corresponding coupled anions. Typical RDFs of the cation-cation, anion-anion, and cation-anion pairs of the two IL systems are presented in Fig. 4. The central phosphorus atoms of the $\left[\mathrm{P}_{66614}\right]^{+}$cations, and the boron atom of the $[\mathrm{BMB}]^{-}$anion are taken as the origins for the intermolecular RDFs from the trajectories of the two simulated systems at different temperatures with a charge scaling factor $s=0.80$. The strong and intense peak located at approximately $0.4 \mathrm{~nm}$ in the P-Cl RDF indicates a well-organized ionic structure with a distinct first coordination shell of chlorine anions around the $\left[\mathrm{P}_{66614}\right]^{+}$cation due to their strong electrostatic interactions in the heterogeneous ionic environment. The boron atom of the $[\mathrm{BMB}]^{-}$anion is not so close to the central
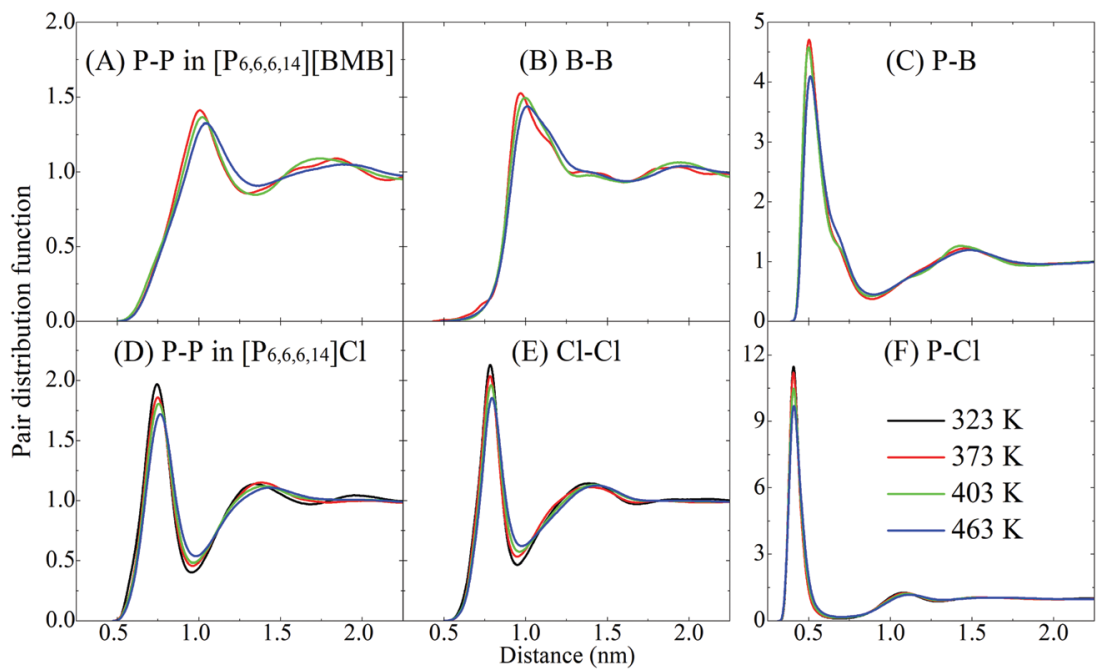

Fig. 4 Radial distribution functions for cation-cation, anion-anion and cation-anion pairs for the studied ILs obtained from the atomistic simulations with a charge scaling factor of 0.80 . The central $P$ atoms in the $\left[\mathrm{P}_{66614}\right]^{+}$cations, and $B$ atoms in the $[B M B]^{-}$anions are taken as reference sites for the calculation of the intermolecular RDFs from recorded equilibrium simulation trajectories. 

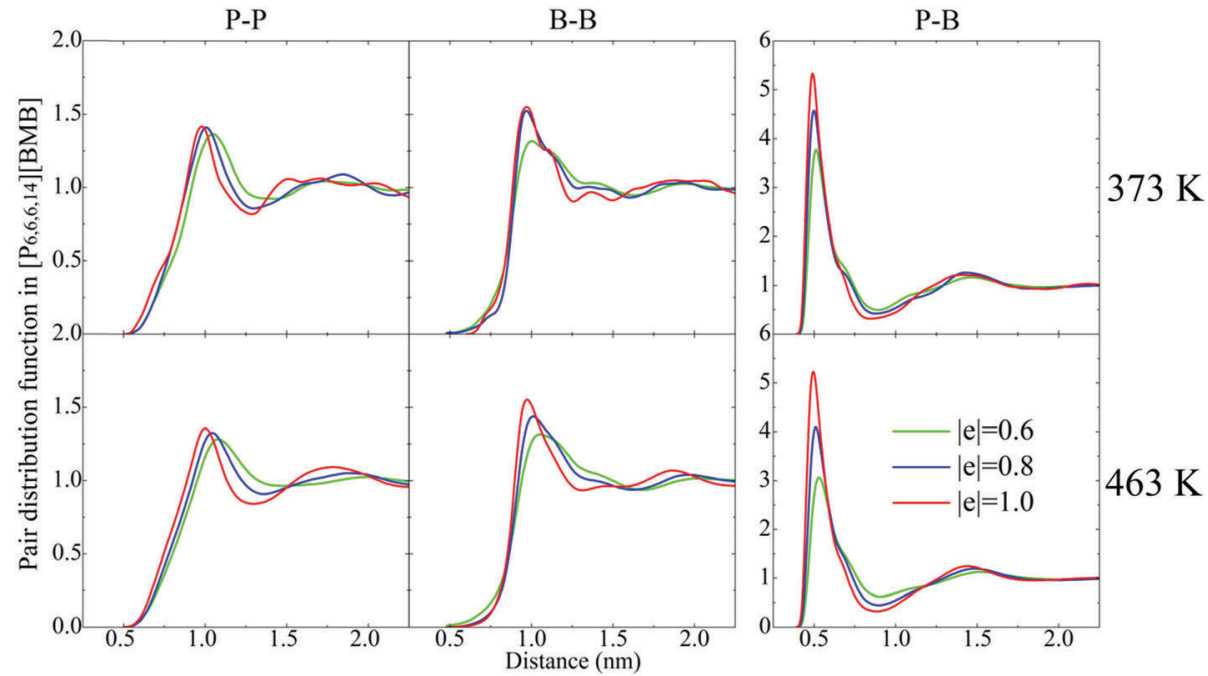

Fig. 5 Effect of the charge scaling factors on typical radial distribution functions of the $\left[\mathrm{P}_{66614}\right][\mathrm{BMB}] \mathrm{IL}$ at 373 and $463 \mathrm{~K}$. The central $\mathrm{P}$ atoms in the $\left[\mathrm{P}_{66614}\right]^{+}$cations, and $\mathrm{B}$ atoms in the $[\mathrm{BMB}]^{-}$anions are taken as reference sites for the calculation of intermolecular RDFs from recorded equilibrium simulation trajectories.

phosphorus atom of the $\left[\mathrm{P}_{66614}\right]^{+}$cation, due to the larger size of this anion. In the simulated $\left[\mathrm{P}_{66614}\right][\mathrm{Cl}]$ system, both the cation-cation and anion-anion RDFs exhibit a sharp first peak at around $0.75 \mathrm{~nm}$, whereas the first peak for the cation-cation and anion-anion RDFs of the $\left[\mathrm{P}_{66614}\right][\mathrm{BMB}]$ system is damped and shifted to a larger distance.

In Fig. 5 we compare the RDFs of $\left[\mathrm{P}_{66614}\right][\mathrm{BMB}]$ for the scaling factors of $1.0,0.80$ and 0.60 . The general feature is that these functions become more diffuse when the scaling factor decreases. The reason for this is that a reduced scaling factor is, to some extent, equivalent to a higher temperature and this leads to a reduced viscosity.

\subsection{Equilibrium vs. nonequilibrium simulations}

For the estimation of the shear viscosity by evaluating the Green-Kubo relation from equilibrium atomistic simulations, a main drawback is that the decay of the time correlation functions of the shear stresses is quite long and it can be difficult to determine whether the upper limit of the time integral of the Green-Kubo relation in eqn (1) is long enough. On the other hand, when nonequilibrium shear flow simulations are performed, the shear rate should be large enough so that the induced shear stress overwhelms the thermal fluctuations in the simulated system. At the same time, the shear rate should be not so large that the relationship between the shear rate and the shear stress becomes nonlinear. However, in practical atomistic simulations, it is often difficult to determine whether the low shear rates fall into the linear regime. This problem can be solved by comparing the shear viscosities estimated from shear flow simulations at low shear rates to those obtained from the Green-Kubo integrals. According to linear response theory, ${ }^{40}$ the shear viscosities obtained from these two methods should be exactly the same at the same thermodynamic state point. This provides a significant consistency test of the two different simulation algorithms used to evaluate the
Table 3 Comparison of the viscosity of $\left[\mathrm{P}_{66614}\right][\mathrm{BMB}]$, estimated from the nonequilibrium shear flow simulations, with the viscosity obtained from the equilibrium simulations using Green-Kubo relations and with different sizes of the simulated systems. These simulations were performed at $423 \mathrm{~K}$ with a charge scaling factor of 0.80 for the $\left[\mathrm{P}_{66614}\right]^{+}$cations and $[\mathrm{BMB}]^{-}$ anions

\begin{tabular}{llcl}
\hline$N$ & $\dot{\gamma} / 10^{6} \mathrm{~s}^{-1}$ & $\eta / \mathrm{mPa}$ s (shear) & $\eta / \mathrm{mPa}$ s (Green-Kubo) \\
\hline 96 & 15.5 & $35 \pm 4$ & $33 \pm 1$ \\
735 & 9.87 & $34 \pm 1$ & $30 \pm 3$ \\
735 & 98.7 & $31.7 \pm 0.4$ & \\
735 & 987 & $18.5 \pm 1$ & \\
6000 & 3.9 & $32 \pm 9$ & \\
6000 & 19.5 & $27 \pm 2$ & \\
6000 & 97.7 & $26 \pm 1$ &
\end{tabular}

viscosity and a test of the correctness of the computer code used to perform the simulation.

We first compare the viscosities of the $\left[\mathrm{P}_{66614}\right][\mathrm{BMB}]$ systems at $423 \mathrm{~K}$ with a charge scaling factor $s=0.80$. The nonequilibrium shear flow simulations were performed at shear rates of $1.55 \times 10^{7}$ and $9.87 \times 10^{6} \mathrm{~s}^{-1}$ for the $\left[\mathrm{P}_{66614}\right][\mathrm{BMB}]$ systems consisting of 96 and 735 ion pairs, respectively. These two shear rates are the lowest shear rates at which we are able to estimate viscosity of $\left[\mathrm{P}_{66614}\right][\mathrm{BMB}]$ without prohibitively long simulations. The results obtained from the nonequilibrium simulations, as well as those obtained from the equilibrium simulations using the Green-Kubo relation, are shown in Table 3. The viscosities estimated from the nonequilibrium shear flow simulations are in good agreement with those obtained from the equilibrium simulations using the Green-Kubo relation within relative errors of less than $10 \%$. In order to show that the results for the computed viscosities are reliable, we display the time integral of the shear stress autocorrelation function from time zero to time $t$ in Fig. 6. It can be seen that it has converged after about $3 \mathrm{~ns}$, demonstrating that the Green-Kubo relation also has converged. An additional nonequilibrium shear flow simulation was performed 


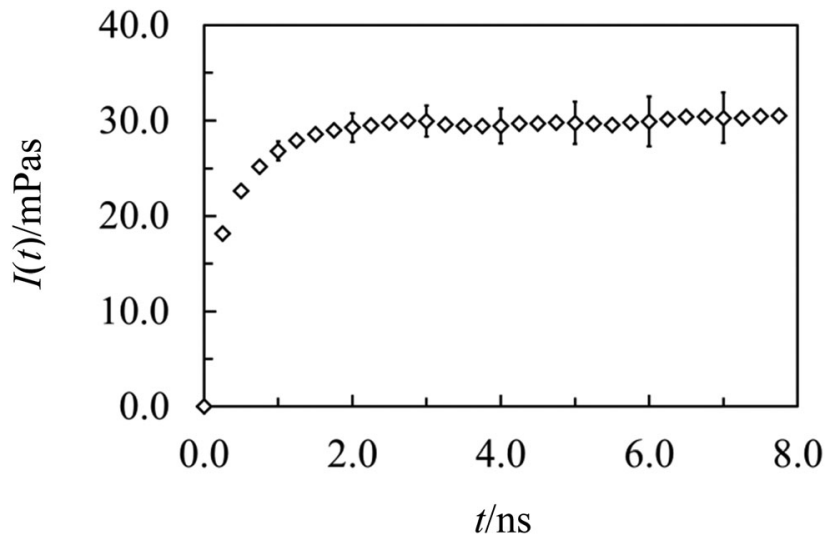

Fig. 6 The time integral of the shear stress autocorrelation function from time zero to time $t$. In the limit of infinite time it is equal to the Green-Kubo integral for the viscosity, eqn (1).

on a very large simulation system consisting of $6000\left[\mathrm{P}_{66614}\right][\mathrm{BMB}]$ ion pairs at shear rates of about $3.9 \times 10^{6} \mathrm{~s}^{-1}$. Although, it is not possible to evaluate the Green-Kubo relations for the viscosities of such large systems with presently available computer resources, the viscosity results estimated from the nonequilibrium shear flow simulations agree very well with those obtained from the smaller simulation systems.

Herein we again verify that the computed viscosities estimated from the smaller $\left[\mathrm{P}_{66614}\right][\mathrm{BMB}]$ simulation system consisting of 96 ion pairs are reliable and such a simulation system is used to further perform nonequilibrium shear flow simulations at $423 \mathrm{~K}$ with a charge scaling factor of $s=0.80$. In Fig. 7, we present the computed viscosities for $\left[\mathrm{P}_{66614}\right][\mathrm{BMB}]$ as a function of the shear rate up to $1.0 \times 10^{10} \mathrm{~s}^{-1}$ for the three different system sizes that we have studied and they agree reasonably well even at higher shear rates. In the nonequilibrium shear flow simulations $\left[\mathrm{P}_{66614}\right][\mathrm{BMB}]$ exhibits non-Newtonian behavior, when the shear rate exceeds $1.0 \times 10^{8} \mathrm{~s}^{-1}$. In Fig. 8, we show the computed viscosities for

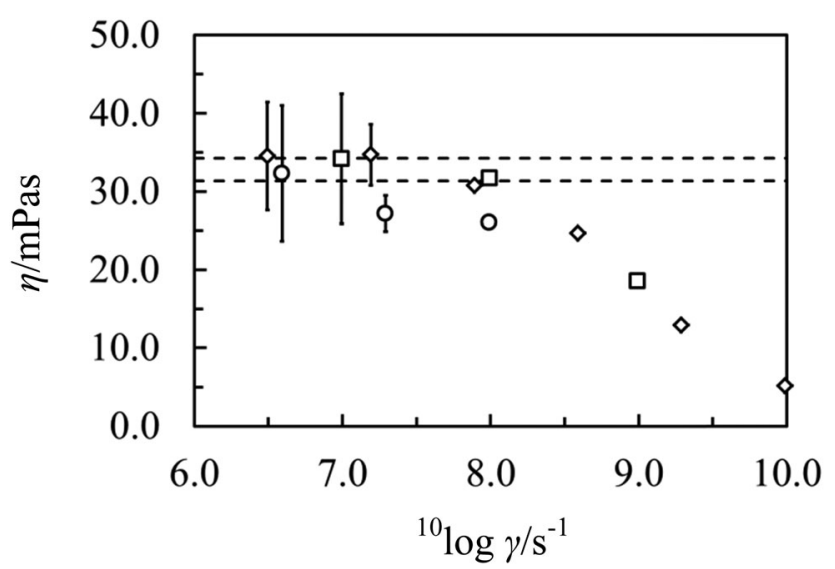

Fig. 7 Viscosity values, $\eta$, at $423 \mathrm{~K}$ for $\left[\mathrm{P}_{66614}\right][\mathrm{BMB}]$ as a function of the shear rate, $\gamma$, obtained from systems consisting of 96 (squares), 735 (diamonds) and 6000 (circles) ion pairs, respectively. The dashed lines mark the limits of the equilibrium Green-Kubo estimates of the viscosity of the smallest system consisting of 96 ion pairs.

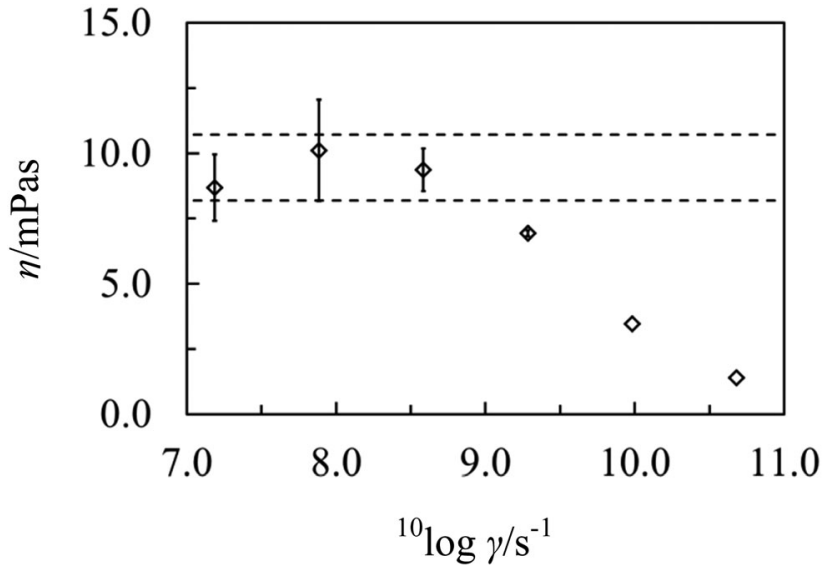

Fig. 8 Viscosity values, $\eta$, for $\left[\mathrm{P}_{66614}\right][\mathrm{Cl}]$ as a function of the shear rate, $\gamma$, obtained from a simulation system consisting of $256\left[\mathrm{P}_{66614}\right][\mathrm{Cl}]$ ion pairs at $423 \mathrm{~K}$. The dashed lines mark the limits of the equilibrium Green-Kubo estimates of the viscosity.

$\left[\mathrm{P}_{66614}\right][\mathrm{Cl}]$ as a function of shear the rate, up to $1.0 \times 10^{11} \mathrm{~s}^{-1}$. Here, the non-Newtonian behavior begins at a shear rate of about $1.0 \times 10^{9} \mathrm{~s}^{-1}$ which is somewhat higher than in the case of $\left[\mathrm{P}_{66614}\right][\mathrm{BMB}]$. This can be rationalised by noting that the molecules of $\left[\mathrm{P}_{66614}\right][\mathrm{BMB}]$ are larger than those of $\left[\mathrm{P}_{66614}\right][\mathrm{Cl}]$. In the non-Newtonian regime, the computed viscosities both for $\left[\mathrm{P}_{66614}\right][\mathrm{BMB}]$ and $\left[\mathrm{P}_{66614}\right][\mathrm{Cl}]$ present an inverse linear dependence on the logarithm of shear rate, i.e. a power law dependence. This is consistent with the behavior of nonpolar alkanes in strong shear fields. Finally, it should be noted that the onset of the nonNewtonian regime appears at such a high shear rate that it is hard to verify the power law dependence from currently available experimental data.

In Table 2 we show the computed viscosities of $\left[\mathrm{P}_{66614}\right][\mathrm{Cl}]$ evaluated from nonequilibrium shear flow simulations at two shear rates of $1.5 \times 10^{7}$ and $7.5 \times 10^{7} \mathrm{~s}^{-1}$, and at three temperatures of 383,423 and $463 \mathrm{~K}$. A slight temperature variation of shear rate in the nonequilibrium simulations is due to the fact that we actually keep the displacement rate constant independently of the temperature when the Lees-Edwards sliding brick boundary conditions are applied. Then the shear rate will vary, because the simulation cell dimensions increase with the temperature. The viscosities from the shear flow simulations are compared with those obtained from the equilibrium simulations using the Green-Kubo relation. It is clearly shown that the agreement between the computed viscosities estimated from the shear flow simulations and those from the equilibrium simulations, is very good. These simulation results indicate that these two methods can give consistent computational results in a rigorous procedure, not only for simple fluid systems but also for complex molecular systems like the phosphonium ILs characterized by distinct microstructural and dynamical heterogeneities.

\section{Conclusion}

We have studied the rheological properties of two ILs composed of a common trihexyl(tetradecyl)phosphonium cation coupled with 
bis(mandelato)borate and chloride anions, respectively, by combining molecular dynamics simulations and experimental measurements. The IL viscosities were evaluated from the equilibrium simulations using the Green-Kubo relation, and, in parallel, estimated from the nonequilibrium shear flow simulations in the linear regime. These two methods give results that agree with each other within the statistical uncertainty as they should according to linear response theory. The dependence of the computed IL viscosity on the simulation system sizes was studied for systems consisting of 96, 735 and 6000 ion pairs. It was found that it was possible to reproduce the experimentally measured viscosities by using a simulation system consisting of 96 ion pairs.

Flat charge scaling factors on the ion charges were considered in the current work to account for the molecular polarizability and charge transfer phenomena between the large molecular ionic species in the phosphonium ILs. The computed viscosities of $\left[\mathrm{P}_{66614}\right][\mathrm{BMB}]$ in the temperature interval of 383-463 K show that the experimental results can be reproduced by scaling factors between 0.60 and 0.80 whereas the corresponding results for $\left[\mathrm{P}_{66614}\right][\mathrm{Cl}]$ in the temperature interval of $343-463 \mathrm{~K}$ can be reproduced by scaling factors between 0.80 and 1.0. This difference is attributed to the fact that the $[\mathrm{BMB}]^{-}$anion is much larger and molecularly more complex than the $[\mathrm{Cl}]^{-}$anion and, therefore, provides more possibilities for polarization and charge transfer through the bonds and ring structures. The computed viscosities of the two ILs follow an Arrhenius-like relationship as a function of temperature whereas the experimentally measured viscosities do not. This deviation is attributed to the temperature dependent polarizability and charge transfer effects.

Using the SLLOD equations of motion it is not only possible to obtain the viscosity in the Newtonian regime but also in the non-Newtonian regime, at high shear rates. Doing this we found that the onset of the non-linear behavior takes place at a shear rate of about $10^{8} \mathrm{~s}^{-1}$ for $\left[\mathrm{P}_{66614}\right][\mathrm{BMB}]$ and at about $10^{9} \mathrm{~s}^{-1}$ for $\left[\mathrm{P}_{66614}\right][\mathrm{Cl}]$ i.e. the onset appears at a lower shear rate for $\left[\mathrm{P}_{66614}\right][\mathrm{BMB}]$. This is logical because the molecules of the latter substance are larger. In the nonlinear regime, the viscosity is a linear function of the logarithm of the shear rate. This behaviour has also been observed for linear and branched alkanes.

More studies are required to fully understand the rheology of the ILs and the interactions and mechanisms responsible for the behavior of the transport coefficients over the wide temperature range where the ionic liquids appear. Systematic molecular dynamics simulation studies, as reported in this work, are valuable, although it is obvious that the nonpolarizable force fields cannot be applied directly in the same way as for ordinary liquids and solvents. Neither can these force fields be transferred from one IL system to another. Due to the large sizes and complexities of the molecular ions and the heterogeneity of the liquid structure with its long-ranged structural correlations, we could not afford to use polarizable simulation schemes as the computations were already very demanding. In research related to industrial applications and products, large-scale computations, as the ones used in this work, are not yet an option, but it would be highly desirable to find fast descriptor-based or equation-of-state modeling methods for the ILs similar to those already existing for transport coefficients of ordinary liquids and solvents.

\section{Conflicts of interest}

There are no conflicts to declare.

\section{Appendix}

One way of illustrating how the charge scaling compensates for the lack of polarizability is to consider the Debye length, $\lambda_{\mathrm{D}}$, given by ${ }^{60}$

$$
\lambda_{\mathrm{D}}^{2}=\frac{\varepsilon_{\mathrm{r}} \varepsilon_{0} k_{\mathrm{B}} T}{2 e^{2} N_{\mathrm{A}} I}
$$

where $\varepsilon_{\mathrm{r}}$ is the dielectric constant, $\varepsilon_{0}$ is the permittivity of vacuum, $e$ is the elementary charge, $N_{\mathrm{A}}$ is Avogadro's number, $I$ is the ionic strength,

$$
I=\frac{1}{2} \sum_{i}^{M} c_{i} z_{i}^{2}=c s^{2}
$$

$c_{i}$ is the concentration of the ionic species, $z_{i}$ is the valence of ion $i$ and $M$ is the number of charged species. The second equality is valid for a two component ionic liquid, where the charges of either species are equal to unity but of opposite sign, $s$ is the scaling factor and $c$ is the concentration. If this ionic strength is inserted in the expression for the Debye length, we obtain

$$
\lambda_{\mathrm{D}}^{2}=\frac{\varepsilon_{\mathrm{r}} \varepsilon_{0} k_{\mathrm{B}} T}{2 e^{2} N_{\mathrm{A}} c s^{2}} .
$$

From this expression it can be seen that the scaling factor to some extent compensates for the lack of polarizability and charge transfer.

The Debye-lengths for the different scaling factors are given in Table 4 for a dielectric constant of unity, since it is a major undertaking to calculate the this constant for ionic liquids. ${ }^{61}$ In order to obtain the correct values of the Debye-length these values must be multiplied by the square root of the dielectric constant. However, since the side of the simulation cell in the smallest system of 96 ion pairs is equal to $5.1 \mathrm{~nm}$, this side is longer than the Debye length provided that the dielectric constant is less than 60 .

Table 4 The concentration, $c$, and the Debye length $\lambda_{D}$ as functions of temperature, $T$, and the charge scaling factor, $s$ for a dielectric constant equal to one

\begin{tabular}{lllll}
\hline$T / \mathrm{K}$ & $c / \mathrm{mol} \mathrm{dm}^{-3}$ & $\lambda_{\mathrm{D}} / \AA(s=1.00)$ & $\lambda_{\mathrm{D}} / \AA(s=0.80)$ & $\lambda_{\mathrm{D}} / \AA(s=0.60)$ \\
\hline 383 & 1.21 & 0.354 & 0.442 & 0.590 \\
403 & 1.19 & 0.366 & 0.457 & 0.610 \\
423 & 1.17 & 0.378 & 0.473 & 0.630 \\
443 & 1.16 & 0.388 & 0.485 & 0.647 \\
463 & 1.14 & 0.401 & 0.501 & 0.668
\end{tabular}




\section{Acknowledgements}

We acknowledge the Knut and Alice Wallenberg Foundation (Project number: KAW2012.0078) and the Swedish Research Council (Project number: 2014-4694) for their financial support. We are grateful to professor Oleg N Antzutkin's group for providing the $\left[\mathrm{P}_{66614}\right][\mathrm{BMB}]$ ionic liquid. The simulations were performed using resources provided by the Swedish National Infrastructure for Computing (SNIC) at PDC, HPC2N and NSC. We also acknowledge PRACE for awarding us access to Hazelhen based in Germany at Rechenzentrum Stuttgart. Finally we are indebted to professor Berk Hess for helping us with the implementation of the Gromacs simulation program.

\section{References}

1 K. Binnemans, Chem. Rev., 2005, 105, 4148-4204.

2 Y. Ji, R. Shi, Y. Wang and G. Saielli, J. Phys. Chem. B, 2013, 117, 1104-1109.

3 A. Filippov, F. U. Shah, M. Taher, S. Glavatskih and O. N. Antzutkin, Phys. Chem. Chem. Phys., 2013, 15, 9281-9287.

4 M. A. Javed, S. Ahola, P. Håkansson, O. Mankinen, M. K. Aslam, A. Filippov, F. U. Shah, S. Glavatskih, O. N. Antzutkin and V. V. Telkki, Chem. Commun., 2017, 53, 11056-11059.

5 Y.-L. Wang, M. Golets, B. Li, S. Sarman and A. Laaksonen, ACS Appl. Mater. Interfaces, 2017, 9, 4976-4987.

6 Y.-L. Wang, Z.-Y. Lu and A. Laaksonen, Phys. Chem. Chem. Phys., 2014, 16, 20731-20740.

7 F. Endres and S. Z. El Abedin, Phys. Chem. Chem. Phys., 2006, 8, 2101-2116.

8 D. R. Macfarlane, N. Tachikawa, M. Forsyth, J. M. Pringle, P. C. Howlett, G. D. Elliott, J. H. Davis, M. Watanabe, P. Simon and C. A. Angell, Energy Environ. Sci., 2014, 7, 232-250.

9 S. Glavatskih and E. Höglund, Tribol. Int., 2008, 41, 934-939.

10 P. K. Cooper, H. Li, M. W. Rutland, G. B. Webber and R. Atkin, Phys. Chem. Chem. Phys., 2016, 18, 23657-23662.

$11 \mathrm{H}$. Li, R. J. Wood, M. W. Rutland and R. Atkin, Chem. Commun., 2014, 50, 4368-4370.

12 N. Hjalmarsson, D. Wallinder, S. Glavatskih, R. Atkin, T. Aastrup and M. W. Rutland, Nanoscale, 2015, 7, 16039-16045.

13 M. A. Ploss, M. W. Rutland and S. Glavatskih, Phys. Chem. Chem. Phys., 2016, 18, 26609-26615.

14 Y. Chen, C. Ye, H. Wang and W. Liu, J. Synth. Lubr., 2003, 20, 217-225.

15 F. U. Shah, S. Glavatskih and O. N. Antzutkin, Tribol. Lett., 2013, 51, 281-301.

16 F. U. Shah, S. Glavatskih, D. R. MacFarlane, A. Somers, M. Forsyth and O. N. Antzutkin, Phys. Chem. Chem. Phys., 2011, 13, 12865-12873.

17 R. Gusain, P. Gupta, S. Saran and O. P. Khatri, ACS Appl. Mater. Interfaces, 2014, 6, 15318-15328.

18 K. Sprenger, V. W. Jaeger and J. Pfaendtner, J. Phys. Chem. B, 2015, 119, 5882-5895.

19 T. Yan, C. J. Burnham, M. G. Del Pópolo and G. A. Voth, J. Phys. Chem. B, 2004, 108, 11877-11881.
20 B. L. Bhargava and S. Balasubramanian, J. Chem. Phys., 2005, 123, 144505.

21 O. Borodin, J. Phys. Chem. B, 2009, 113, 11463-11478.

22 O. Borodin and G. D. Smith, J. Phys. Chem. B, 2006, 110, 11481-11490.

23 O. Borodin, G. D. Smith and H. Kim, J. Phys. Chem. B, 2009, 113, 4771-4774.

24 C. Rey-Castro and L. F. Vega, J. Phys. Chem. B, 2006, 110, 14426-14435.

25 T. Köddermann, D. Paschek and R. Ludwig, ChemPhysChem, 2007, 8, 2464-2470.

26 M. S. Kelkar, W. Shi and E. J. Maginn, Ind. Eng. Chem. Res., 2008, 47, 9115-9126.

27 M. S. Kelkar and E. J. Maginn, J. Phys. Chem. B, 2007, 111, 4867-4876.

28 W. Zhao, F. Leroy, S. Balasubramanian and F. MüllerPlathe, J. Phys. Chem. B, 2008, 112, 8129-8133.

29 L. J. A. Siqueira and M. C. C. Ribeiro, J. Phys. Chem. B, 2009, 113, 1074-1079.

30 N. T. Van-Oanh, C. Houriez and B. Rousseau, Phys. Chem. Chem. Phys., 2010, 12, 930-936.

31 H. Liu and E. Maginn, J. Chem. Phys., 2011, 135, 124507.

32 Z. Liu, T. Chen, A. Bell and B. Smit, J. Phys. Chem. B, 2010, 114, 4572-4582.

33 X. Zhong, Z. Liu and D. Cao, J. Phys. Chem. B, 2011, 115, 10027-10040.

34 E. Choi, J. G. McDaniel, J. Schmidt and A. Yethiraj, J. Phys. Chem. Lett., 2014, 5, 2670-2674.

35 M. Chen, R. Pendrill, G. r. Widmalm, J. W. Brady and J. Wohlert, J. Chem. Theory Comput., 2014, 10, 4465-4479.

36 Y. Zhang, A. Otani and E. J. Maginn, J. Chem. Theory Comput., 2015, 11, 3537-3546.

37 N. Voeltzel, A. Giuliani, N. Fillot, P. Vergne and L. Joly, Phys. Chem. Chem. Phys., 2015, 17, 23226-23235.

38 S. Bair, C. McCabe and P. T. Cummings, Phys. Rev. Lett., 2002, 88, 058302.

39 C. McCabe, S. Cui and P. T. Cummings, Fluid Phase Equilib., 2001, 183, 363-370.

40 D. J. Evans and G. Morriss, in Statistical Mechanics of Nonequilibrium Liquids, ed. D. J. Evans and G. Morriss, Cambridge University Press, Cambridge, 2nd edn, 2008.

41 S. Sarman and A. Laaksonen, J. Comput. Theor. Nanosci., 2011, 8, 1081-1100.

42 Y.-L. Wang, S. Sarman, S. Glavatskih, O. N. Antzutkin, M. W. Rutland and A. Laaksonen, J. Phys. Chem. B, 2015, 119, 5251-5264.

43 T. G. Youngs and C. Hardacre, ChemPhysChem, 2008, 9, 1548-1558.

44 R. Ishizuka and N. Matubayasi, J. Chem. Theory Comput., 2016, 12, 804-811.

45 M. Salanne, Phys. Chem. Chem. Phys., 2015, 17, 14270-14279.

46 K. Bica, M. Deetlefs, C. Schröder and K. R. Seddon, Phys. Chem. Chem. Phys., 2013, 15, 2703-2711.

47 J. J. Molina, S. Lectez, S. Tazi, M. Salanne, J.-F. Dufrêche, J. Roques, E. Simoni, P. A. Madden and P. Turq, J. Chem. Phys., 2011, 134, 014511. 
48 Y. Gu and T. Yan, J. Phys. Chem. A, 2012, 117, 219-227.

49 C. Millot, A. Chaumont, E. Engler and G. Wipff, J. Phys. Chem. A, 2014, 118, 8842-8851.

50 L. Huang and B. Roux, J. Chem. Theory Comput., 2013, 9, 3543-3556.

51 T. I. Morrow and E. J. Maginn, J. Phys. Chem. B, 2002, 106, 12807-12813.

52 C. M. Breneman and K. B. Wiberg, J. Comput. Chem., 1990, 11, 361-373.

53 C. I. Bayly, P. Cieplak, W. Cornell and P. A. Kollman, J. Phys. Chem., 1993, 97, 10269-10280.

54 D. Roy and M. Maroncelli, J. Phys. Chem. B, 2010, 114, 12629-12631.
55 Y.-L. Wang, S. Sarman, B. Li and A. Laaksonen, Phys. Chem. Chem. Phys., 2015, 17, 22125-22135.

56 B. Hess, C. Kutzner, D. Van Der Spoel and E. Lindahl, J. Chem. Theory Comput., 2008, 4, 435-447.

57 S. Gabl, C. Schröder and O. Steinhauser, J. Chem. Phys., 2012, 137, 094501.

58 J. C. Araque, J. J. Hettige and C. J. Margulis, J. Phys. Chem. B, 2015, 119, 12727-12740.

59 S. Kim, S.-W. Park and Y. Jung, Phys. Chem. Chem. Phys., 2016, 18, 6486-6497.

60 P. Atkins and J. de Paula, Atkins' Physical Chemistry, Oxford University Press, Oxford, 2010.

61 C. Schröder, T. Rudas and O. Steinhauser, J. Chem. Phys., 2006, 125, 244506. 